\title{
OBSERVATIONS ON STERTOR,
}

\author{
AND ON THE \\ VARYING CONDITIONS UPON WHICH IT IS DEPENDENT, \\ WI'TH THE TREATMENT NECESSARY TO ITS RELIEF. \\ BY \\ ROBERT I. BOWLES, M.R.C.S. \& L.S.A., \\ OF FOLKSTONE. \\ COMMUNICATED BY \\ CASAR H. HAWKINS, F.R.S., \&c.
}

Received Nor. 21st, 1859.-Read Jan. 24th, 1860.

IN order to make my subject clear, I am necessarily led to speak of obstructions about the throat in connection with stertor; how far these obstructions are due to the position of the body, and how they may be removed by altering that position.

The cause of the loud and deep sound in the throat to which the name "stertor" has been applied, does not appear to have received the attention it deserves. : From numerous experiments $I$ have arrived at the conclusion that in the majority of instances it arises from one of three conditions : from paralysis of the velum palati; from the paralysed tongue falling back in the throat; and from the presence of mucus in the pharynx and air-passages.

In the winter of 1855 and 1856 I was engaged, in conjunction with Dr. Edward Long Fox, of Bristol, and Mr. Charles Hunter, of Wilton Place, upon some experiments for the late Dr. Marshall Hall, to determine the amount of air 
which could be respired in the dead subject by compression and relaxation of the chest. In attempting this we repeatedly found that at one time compression failed to expel any air from the chest, and at another, after air had been expelled, it would not re-enter on removing the pressure. On opening the mouth and searching for a cause, it was found that the tongue had fallen back and blocked up the cavity of the pharynx. The tongue was accordingly drawn forward and fixed between the incisor teeth, but still we found that on one body the experiment succeeded well in producing respiration, in another it failed. This time the tongue was no obstacle, but the pressure on the chest and abdomen had thrown up some of the contents of the stomach into the pharynx, which entirely prevented the current of air from passing into or out of the chest. This induced us to try to produce respiration in such positions that anything in the pharynx would fall from rather than towards its posterior wall; the consequence was, that we obtained the most invariable and favorable results by the method of artificial respiration now known as that of Marshall Hall.

In a paper communicated to the Medical Society of St. George's Hospital in 1856, great stress was laid on the influence of the position of the body over the tongue in the half-drowner patient; and it was pointed out how necessary it became to pay the greatest attention to a fact which at first sight appeared of little importance. Subsequent to the meeting I received from Mr. Harvey, one of the members of the Society, a letter on the subject, of which the following is an extract :

"I am glad to find you lay so much stress on the tongue's position producing apncea in persons that have been rendered insensible. One of the first persons I ever saw on the operating-table nearly died from this cause during the operation, under the influence of chloroform. Mr. Syme, who was operating, fortunately guessed what was the cause of the sudden asphyxia, and drew the tongue forward with a pair of forceps, and the patient presently recovered." 
Mr. Harvey's case strikingly illustrates that the tongue may be a cause of apnoa in other circumstances than those of drowning. The following cases illustrate another phase of this subject :

Case 1.-In November, 1857, I was called to see an old lady who had been attacked with sudden sickness whilst talking to some friends; faintness followed, with an inability to express herself, though she was to a considerable extent conscious. She was put to bed, and kept quiet; mustard poultices were applied to the epigastrium and nape of neck, and hot bottles to the feet. In an hour or two reaction commenced, and at the same time coma and stertorous breathing set in, which steadily increased in intensity until my second visit, at which time this condition had lasted about three hours. Her pulse was now full and excited, but as she was seventy-three years of age, and had already suffered from two or three attacks of paralysis, no severe measures were adopted. The skin was hot, and covered with a profuse perspiration; the face was swollen and livid, and the mouth half open; the pupils were contracted and insensible to light, and the eyeball to touch. The stertor was very deep, and the breathing laboured. Whilst I was at the bedside of my patient she was very nearly suffocated, from the stomach ejecting a portion of its contents into the throat. I at once turned her on her side and emptied her mouth, when she not only breathed freely, but without the slightest stertor. I was somewhat surprised, but proceeded to place her in what appeared to be the most comfortable positicn, on her back. Immediately when I did so, her respiration suddenly ceased, and she convulsively struggled for breath. Supposing it to be the tongue which had choked her, I thrust my finger into the mouth, and pressed it forward, when the breathing again became easy and free from stertor. The patient was now comfortably laid on her side; and until her death, which took place two days afterwards, the stertor never recurred when she was in that position, but the respiration continued as naturally as if she slept. To convince 
myself that this was due to position, I several times turned her on her back for a few moments, and invariably, when I did so, the stertor was as marked as ever. The lateral position had another advantage, for it allowed the saliva and mucus to drain away instead of remaining in the bronchial tubes and giving rise to "mucous stertor," and thus offering another impediment to the respiration. Other changes occurred coincidently with the change of position; but how far they depended upon it, future experience must decide. The pulse became calmer; the skin cooler and less bedewed with moisture; the eye sensible to the touch; and general sensation returned to such an extent that the patient evinced signs of discomfort at the removal of a mustard poultice. Nerertheless, whenever the supine position was adopted, the stertor, difficulty of breathing, lividity of face, and other unfavorable symptoms, returned in full force. It was easy to observe, that as the position was changed, the paralysed tongue gravitated downwards.

This case suggests many important points for consideration:-lst. That the paralysed tongue may, under certain circumstances, cause even death by suffocation ; for I believe this patient would have died if I had not been present when the tongue fell so suddenly back, and when the fluid was ejected from the stomach into the throat. 2dly. That stertor arises from the tongue falling back in the supine position of the body, thus offering a serious impediment to the respiration; since it required greater muscular force to breathe with stertor than when the respiration was noiseless. $3 \mathrm{dly}$. The mucus (another respiratory impediment) drained away when the patient was on her side. 4.thly. The great improvement of the general symptoms occurring upon the establishment of easy breathing in the lateral position : pulse, skin, and sensation all undergoing material alterations for the better.

CASE 2.-G. T-, a gentleman 65 years of age, became comatose from exhaustion supervening, upon extensive sub- 
acute inflammation of emphysematous lungs. When I entered the room, he was lying on his back, with his mouth open, breathing stertorously, though not to a great extent. On looking into the mouth, the soft palate appeared remarkably small and shrivelled, so that it did not come very decidedly in contact with the tongue, even though partially paralysed. Whilst I was standing at the bedside, my patient suddenly ceased to breathe, became very livid in the face, struggled convulsively, and then became rigid. My recollection of the first case induced me to turn this patient on his side, when he instantly recovered with a long groan, and then breathed quite naturally. He was turned on his back again; stertor returned, and he became choked from a quantity of bronchial secretion. The lateral position was resumed, the sputa drained away, and he now breathed quite easily until his death, which took piace about half an hour afterwards.

Case 3.-H. J. L-, a clergyman, about 60 years of age, subject to slight epileptic scizures twice a year, was seized, on May 4th, 1858, at ten in the morning, with a fit of the usual character. The attack commenced with a scream, and lasted but a few minutes, though the patient was some time before he fully recovered. At eight o'clock in the evening of the same day, he was again attacked whilst walking across the hall of the hotel at which he was staying, When I arrived about ten minutes afterwards, I found him lying on his back, with a deep cut orer the left eye, breathing stertorously, and with much labour. The mouth was open, the face lighly congested, the skin hot and perspiring, and the pulse full and sharp, but not accelerated. He was quite insensible to external impressions. He was turned on his side sufficiently for the tongue to fall forward; the stertor instantly gave place to easy, natural breathing; the congestion of the countenance gradually disappeared, and in less than one minute he began to evince signs of recovery, and almost as quickly the pulse became weak and small, and the skin cool. He went to sleep for a few minutes, and on awaking 
was rendered almost frantic by pain in his head. From this he slowly recovered, and in a few days was convalescent. I should mention that there was a loud bruit at the apex of the heart, and that a small pulse was common to him.

Case 2 merely gave further evidence of the danger of the supine position of the body from paralysis of the tongue and the accumulating bronchial secretion; but in Case 3 it would appear that the change of position was in some measure the cause of his speedy recovery. In this case, too, as in Case 1, the skin and pulse altered in character as the stertor ceased. It is in such a case as this that I can imagine "position" to be a curative as well as a palliative measure. Here was a delicate man, subject to epilepsy and disease of the mitral valves. Supposing the breathing to have gone on with great labour, and the lungs to have become more and more congested, and consequently the brain and heart also, one or other, or both of these vital organs, might have suffered, and death ensued.

Case 4.-Mrs. P-, æt. 46, after suffering twelve days from continued fever, was seized on the evening of August 27 th, 1858, with a severe rigor, which was soon followed by what was supposed to have been a very deep sleep. I was called to see her at two in the morning of the 28th, when I found her lying upon her side, in a state of deep coma, with her mouth half open. There was no stertor; the skin was warm and bedewed with moisture, and the pulse was almost imperceptible. I endeavoured to administer a little stimulant, and for that purpose turned the face upwards, when I soon perceived a harshness in the breathing, from obstruction in the back of the throat. By allowing the head to remain in this position a few minutes, the obstruction became greater at each inspiration, and stertor was the result. A little fluid was now introduced into the mouth with a teaspoon, which almost choked my patient; but on turning her on her side again, the fluid drained away, and the stertor ceased. In about an hour afterwards she sank. 
So prostrate was this poor woman from fever, that I believe, had much effort been required to overcome the impediment to the respiration, from the time coma first set in, she would not have survived as long as she did.

Besides the cases now related, a medical friend informs me that, on my suggestion, he adopted the lateral position in a case of stertor from apoplexy, and that the stertor immediately ceased. The man was bled, and soon recovered from the comatose condition. My friend also adopted this method with a child suffering from convulsions. When he first saw the child, it was frothing at the mouth, and the respiration seemed much impeded. He turned it on its side, and before he had time to apply other remedies, the child began to evince signs of recovery. I do not assume in these cases, or in Case 3, that change of position was the cause of recovery: it may have assisted to produce the happy result, or it may merely have been a coincidence. I simply state the facts as they occurred, and aroid inductions for the present.

Meanwhile, I propose roughly to consider the anatomy of the parts concerned in stertor, and to show how and why stertor is the consequence of the supine position of the body, and why it differs in character in different cases. I must also relate a few experiments undertaken with the object of further illustrating the views herein entertained.

It is well known that the cavity of the pharynx, having a fixed boundary only posteriorly, may have its capacity materially affected by the ever-varying position of its sides, of the soft palate, the tongue, and the larynx. The larynx and sides of the pharynx have, I believe, little connection with the cause of stertor. I shall therefore not further refer to them. The tongue being attached to the lower jaw by its mucous membrane and its muscles, has its relations with surrounding parts altered as the mouth is opened or closed.

When the mouth is closed, the ramus of the jaw forms nearly a right angle with the spinal column, from which the symphysis is then at its greatest distance. When the mouth 
is opened by the dropping of the jaw, the symphysis describes the arc of a circle, and approaches more nearly to the spine, where the posterior wall of the pharynx is attached. (See Drawing at the end of the paper, fig. 1.) The tongue having its chief attachment to the symphysis, would consequently be drawn away from the pharynx in the former case, and allowed to rest in contact with it in the latter. This, if not true in every case, is at least true in some, as the plate demonstrates. (See fig. 2.)

The dissection from which the drawing was taken was made with great care, so as not to displace the parts more than was necessary to give a fair view of the base of the tongue and the epiglottis. The two sketches, with the open and closed mouth, were kindly taken for me on the spot by my friend Mr. Fitzgerald, and they very accurately represent the parts as they appeared at the time. When the mouth was open and the subject on its back, the road to the larynx was completely obstructed, in consequence of the tongue resting in contact with the back of the pharynx; but the obstruction could be removed by hooking the tongue forward. When the mouth was closed, the tongue was lifted from the pharynx, leaving ample breathing space. But even with the closed mouth, if the chin were much bent on the sternum, the base of the tongue was almost in contact with the back of the pharynx.

It would seem from this dissection that the muscles of the tongue attaching it to the symphysis of the jaw, are too short to admit of the base of the tongue reaching the back of the throat when the mouth is closed. The purpose served by such an arrangement would appear to be to keep the base of the tongue away from the back of the throat during deglutition. In the act of deglutition, the mouth is firmly closed, and the genio-hyoid and genio-hyo-glossi muscles taking their fixed point from the now immoveable symphysis, draw the body of the tongue upwards and forwards, allowing sufficient room for the morsel of food to travel easily through the cavity of the pharynx. Were these muscles of greater length, the base of the tongue 
might become a source of inconvenience, if not of danger. The dissection, besides demonstrating the respective positions of the tongue with the open and closed mouth, suggests the necessity of caution being used in raising the head with pillows ; for if the head be too much bent forward on the chest, the tongue may lie in dangerous proximity to the pharynx, even if the mouth be closed.

\section{The Different Characters of Stertor.}

From having observed the snoring of sleep sometimes take place through the nostrils with the closed mouth, and sometimes through the open mouth, and having also observed differences in the apoplectic stertor, I endeavoured to analyse the particular causes of each of these varieties by some experiments on myself, and by clinical observation.

I placed myself as nearly as possible in the condition of an apoplectic patient, by paralysing my tongue, or rather by allowing it to assume its own position by gravitation. After a little practice, and with some care, I succeeded in effecting this, and found-lst. That it required much more voluntary effort to snore through the nose and breathe stertorously through the mouth in the upright and prone positions than in the recumbent and supine, and that the greatest impediment to the respiration occurs when the jaw is dropped and the mouth most widely open. 2dly. The deep, vibrating snore of sleep, with the closed mouth, depends upon the tongue pressing the velum palati upwards and backwards, thus narrowing the opening between the nose and pharynx, the velum vibrating as the air rushes through. Sometimes, when the mouth is partially open, the soft palate drops upon the tongue, and vibrates as the air rushes between it and the tongue. To either of these noises in. which the soft palate is concerned $I$ propose, for the sake of distinction, to apply the name of "palatine stertor." 3dly. The harsh, sharp, husky noise, occurring in apoplexy, with the mouth wide open, always arises low down in the pharynx, and depends upon 
the narrowing of the passage between its posterior wall and the base of the tongue. By holding a looking-glass before the face whilst producing this noise, it can readily be seen that the soft palate is in no way concerned in it. In all the cases recorded in this paper this was the nature of the stertor which existed, and as it arises solely in the pharynx, I have given it the name of "pharyngeal stertor." 4thly. There is a variety of stertor in apoplexy which may be named "mucous stertor," as it depends upon the presence of mucus in the bronchial tubes. So common is this, either alone or in combination with one or other of the preceding varieties, that in 'Hooper's Medical Lexicon' it is stated, that "apoplectic stertor appears to depend upon the presence of mucus in the air-tubes," as though it were always the cause.

Of these three different varieties of stertor, that which is named pharyngeal is far the most dangerous, for the greater the paralysis the more effectually does the tongue block up the passage, and each inspiration only adds to the distress by sucking the tongue lower down into the pharynx. Its danger is, however, not due alone to the gradual impediment it offers to the respiration, but also to the fact that at any moment this impediment may be absolute, and cause death by suffocation. This has been before referred to in the remarks upon Case 1.

We may observe a similar example in the snoring sleeper, who, after drawing several inspirations with evident effort, at last comes to a sudden check from having been gagged by the tongue falling back. This will rouse him from his stupor, and he will then sleep on quietly until the same thing recurs from his deepening sleep.

This subject gave rise in my mind to the question-Is the breathing in deep coma invariably carried on through the mouth? I once thought that such was the case. In the four cases related it was so, as well as in some cases of apoplexy which I witnessed at a former period. But the following case is one in which the breathing was carried on through the nostrils. 
Case 5.-W. B-, æt. 41, had suffered for two years from numbness and tingling of the extremities of the right side, and was now (August 20th, 1858) under treatment for a supposed commencing disease of the retina. He had been suffering much from anxiety for some time past, and was unusually drowsy in the daytime. On August 20th, after eating a hearty breakfast, he was seized with paralysis of the right side. When I arrived he was able to speak indistinctly, though not to make use of the words he wished. The face was drawn to the left side, and the tongue pushed to the right when protruded; the pupils were natural. In a few minutes he ejected a large quantity of food from his stomach, and became covered with perspiration. He was conveyed to bed, but before he could be undressed he was partially sick several times. He soon commenced snoring with his mouth closed, and was now unable to answer questions; his pulse was soft and feeble, and his skin clammy. I turned him on his side, and though the snoring altered in character, it did not cease, but the impediment to the breathing was less. The mouth was firmly closed, but by means of a spoon I was able to open it slightly, and found the tongue in contact with the incisor teeth, and closely applied to the palate. By introducing the spoon further into the mouth, and pressing on the tongue, all snoring at once ceased; when, however, the mouth was allowed to close, it continued as before. Medicines could not be swallowed, and in about half an hour he breathed his last, quietly and without a struggle.

Remarks.-Snoring in this case continued even in the lateral position. The spasmodic closure of the jaw had pressed the tongue upwards and backwards, carrying with it the soft palate. The snoring was, however, soft, and slight, and was but a very partial impediment to the respiration.

But I an inclined to believe that the peculiar apoplectic condition giving rise to spasmodic closure of the jaw and "palatine stertor" is exceptional ; and that the proposition that " it is the rule for persons in coma to breathe through 
the mouth" is a true one, and for this reason : the muscles of the jaw being paralysed, the jaw drops and opens the mouth; whereas the dilators of the nostrils being also paralysed, the alæ nasi are forcibly drawn by the in-going air towards the column of the nose, and thus close the orifice of the nares altogether. This can be illustrated by the act of "sniffing;" but the following is a case in point:

Whilst watching the dying moments of a poor man who was comatose from hæmorrhage from an ulcer of the stomach, I observed that he breathed with evident and increasing effort, from the alæ nasi flapping towards the centre column during inspiration. With my fingers I kept the nares freely opened, and the breathing became so easy that $I$ have reason to think that his life was prolonged for some little time by these means.

\section{General Reflections.}

Having now given in detail what evidence $I$ possess in favour of the foregoing views on "stertor" and the "influence of position in certain morbid conditions of the system," I have to add a few remarks on the cases to which this subject appears to apply, and more particularly in reference to apoplexy and its treatment.

Since the days of Abercrombie so much light has been thrown on the different causes of apoplexy, that his division of the primarily apoplectic (where no apparent change had taken place in the brain) may be broken up into several subdivisions. It is now fully acknowledged that many of these cases depend primarily on some disease of the heart or its valves, upon aneurism, upon atheroma, upon engorgement of the pulmonic circulation, and upon uræmia. Consequent upon these great advances in pathology is a total change of treatment, for it is but seldom that we now hear of that indiscriminate and enormous bloodletting which is detailed in Dr. Abercrombie's work on diseases of the brain. In his time, age, sex, or condition had little influence over 
treatment; so long as apoplexy existed, bloodletting was considered the chief, almost the only, remedy.

Since, then, it is admitted that apoplexy may result (and very commonly does result) from an alteration in the state of the general circulation, I conceive that anything. which would mischievously affect the circulation during the apoplectic attack would militate very considerably against the chance of the patient's final recovery. Let me suppose a case. A man with disease of the heart or large vessels, on making some unusual effort, falls down in a fit; he is taken up, and laid on his back in bed. Stertor at once commences; if it be not relieved, the respiration becomes impeded, congestion of the lungs takes place, then of the right side of the heart, then of the jugulars and of the brain. And now another direct source of apoplexy exists-increased congestion of the brain, and this, too, with unaërated blood, itself a poison sufficient to destroy life. As long, then, as the stertor is allowed to continue, the mischief increases, and remedies will be of little avail. But suppose that when the man is taken up in a fit of apoplexy he is laid in such a position that his respiration cannot be impeded, that the blood may flow steadily on in its course; that he is placed in such a position, in short, that nature will meet with no impediment in her attempt to right the temporary disturbance which a sudden effort brought about. Then, I think, we may fairly hope for a better issue, if judicious aid be afforded by means which the urgency of the case may render necessary. This especially applies to cases of congestion dependent upon, or complicated with, atheroma; the danger would then be doubly great, for the increased congestion might be more than the diseased cerebral vessels could bear; they might give way, and the case would become one of sanguineous apoplexy.

In the second class of cases of Dr. Abercrombie (those not primarily apoplectic), where the symptoms depend upon an effusion of blood in the brain, if there be any hope, it must at once be forfeited if the respiration be impeded, since this would increase the congestion of the cerebral vessels 
and cause the blood to make its way out of those which were ruptured with greater force. The same would apply to laceration of any of the sinuses or vessels of the brain from fracture of the skull. I remember to have seen a boy who had fallen from a bridge brought home in a state of syncope. Extensive simple fracture of the vault of the skull was detected. He was laid on his back on a table, and as reaction commenced he fell into a state of coma, with stertorous breathing. In a short time the symptoms became so urgent that trephining was performed, whereupon a large quantity of blood made its escape with considerable force. The boy was relieved, but died three weeks afterwards.

On examination after death, there was found a large quantity of clotted and fluid blood on the surface of the brain, the superior longitudinal sinus was lacerated, and the substance of the brain much injured.

In a case of this kind, to impede the onward current of blood through the jugulars must be the first step towards death, for the blood would make its way where there was least resistance, through the wound of the sinus. If, however, there were no obstruction in its natural course, we may fairly presume that it would follow that rather than turn aside through an opening in which, perhaps, a coagulum had already formed.

Such, then, are the dangers which may result from a persistence of stertor in apoplectic and similar conditions; but there remain the other and more immediate dangers of the supine position. 1st. Fluids or other foreign matters may make their way into the larynx from the mouth or the stomach. This I had repeated opportunities of observing in the dead subject when engaged on experiments for the late Dr. Marshall Hall ; but that it is a real danger in the living man is placed beyond doubt by a remarkable case which is related in the 'Medical Times' of April 23d as having been under the care of Dr. Bristowe. A drunken man died from symptoms of suffocation. At the autopsy some of the contents of the stomach were found in the bronchial tubes. Further, my friend, Mr. Bateman, of Folkstone; informs me 
that during his attendance on a case of delirium tremens, the patient, in an attack of vomiting, became suddenly asphyxiated, and died. No post-mortem examination was made, but it seemed probable that some foreign matter had entered the larynx, and caused spasm of the glottis and death. 2dly. The falling back of the tongue, causing sudden and complete apnœa. This may occur in all cases of paralysis, whether from syncope, apoplexy, concussion of the brain, chloroform poisoning, suffocation from carbonic acid, or drowning. I have seen imminent danger from this cause, but am unable to record a death arising from it, though this may be a more common occurrence than we are at present aware of ; for if a disaster arise from any of the above causes, the patient is, I believe, invariably laid flat on the back.

As an appendix to this subject, and in conclusion, it will be interesting to relate the following case, which has recently been forwarded me by Mr. Lewis (Medical Superintendent of the Cheshire and Chester Lunatic Asylum), to whom I wrote many months ago on this subject :

"Seth Yarwood, a man with a very peculiar formation of head, the subject of ambitious monomania of two and a half years' standing. He first had an epileptic fit, which was of a very severe character, on the 21st of November, 1858, and another on the 10th of December following. They did not recur until June 3d of the present year, when he had one; and two more, one on the 8th and one on the 10th of July:

"On the 10th of August, at 4 p.m., he had four fits, of a very severe nature, in quick succession, three occurring in less than five minutes and the fourth in about a quarter of an hour. When seen, he was in a state of profound coma; pupils contracted and insensible; pulse small, weak, and irregular; surface pale, with cold, clammy perspiration; breathing irregular, slow, and extremely laborious; the stertor was very marked; very little air seemed to pass into the lungs; the cheeks were puffed out during expiration. He was placed well over on his side, and propped up so that 
he remained about half way between the lateral and prone positions. There was an immediate flow of saliva from the mouth, and this was followed by a considerable quantity of sanio-mucous fluid. The breathing instantly became more free, and in less than a minute all stertor had ceased; the other symptoms gradually subsided, and in an hour's time, the bowels having been freely acted on by two drops of croton oil, there was a partial return of consciousness. The next morning he refused to stay in bed, and though dull and stupid, was moving about more or less all day."

Remarks.-The symptoms at the time were considered as rendering a fatal termination more than probable; whether such was really the case is immaterial. The sudden subsidence of the symptoms on the change of posture must, I think, indisputably prove that the cerebral congestion was relieved by it ; and, further, we may conclude that the change in posture rendered a fatal termination less probable than if posture had not been attended to.

\section{EXPLANATION OF PLATE.}

The dissection from which the drawings were taken was made on the body of a woman, æt. 60, who died of cancer of the uterus. Great care was taken not to displace the parts more than was necessary to give a fair view of the base of the tongue and epiglottis. The sketches were taken with the subject lying on its back on the table.

Fig. 1. Showing the position of the tongue with the mouth closed.

Fig. 2. Showing the position of the tongue with the mouth open. 


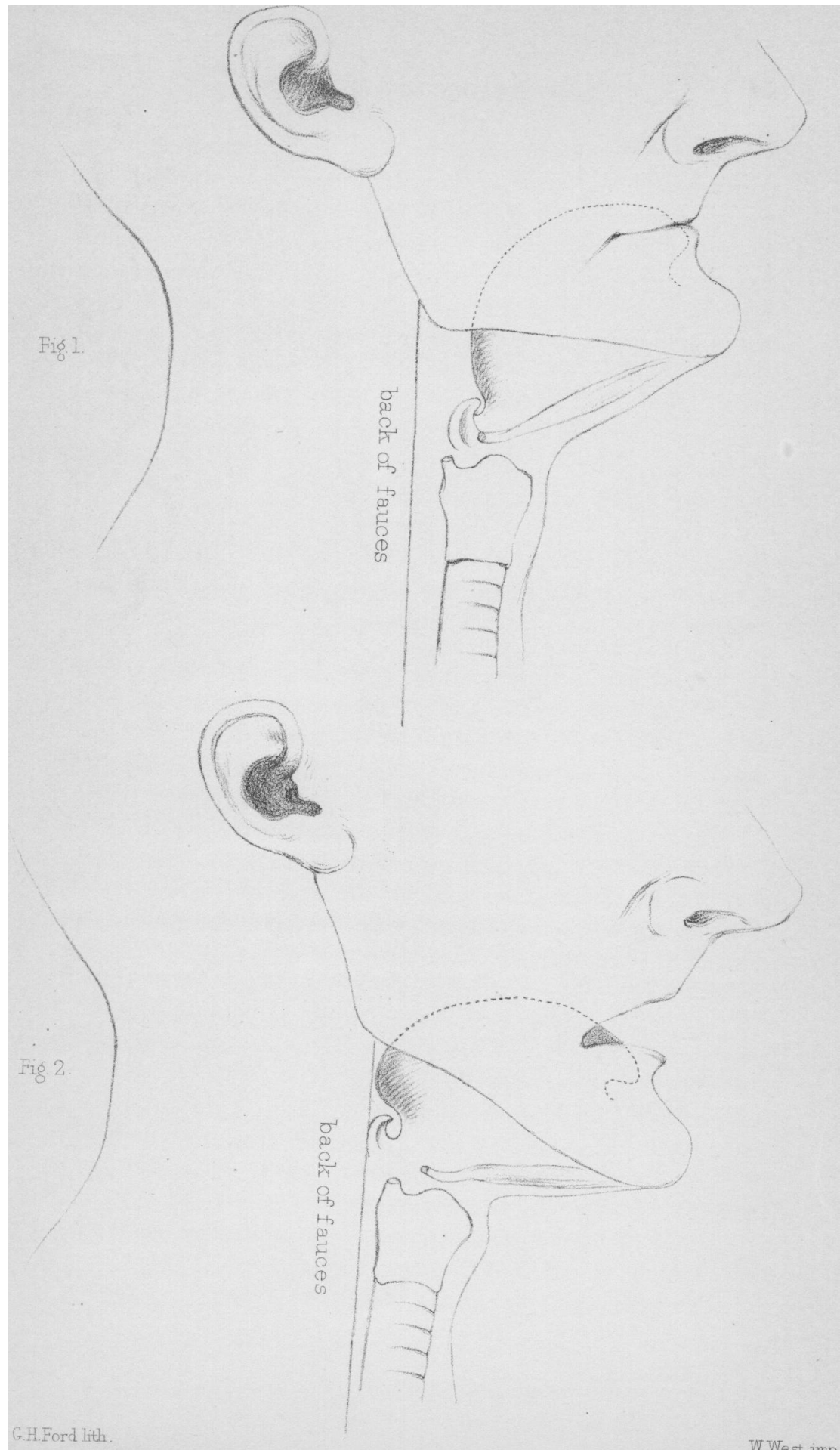

